\title{
From Medical School to Residency
}


Springer Science+Business Media, LLC 
Roger P. Smith, M.D.

Professor and Program Director Director of Ambulatory Care

Department of Obstetrics and Gynecology University of Missouri at Kansas City-Truman Medical Center Kansas City, Missouri, USA

\title{
From Medical School to Residency
}

\author{
How to Compete Successfully \\ in the Residency Match Program
}

With 57 Illustrations 
Roger P. Smith, M.D.

Professor and Program Director

Director of Ambulatory Care

Department of Obstetrics and Gynecology

University of Missouri at Kansas City-Truman Medical Center

2301 Holmes Street

Kansas City, MO 64108

USA

Library of Congress Cataloging-in-Publication Data

Smith, Roger P. (Roger Perry), 1949-

From medical school to residency : how to compete successfully in the residency match program / Roger P. Smith.

p. $\quad \mathrm{cm} .-$

Includes bibliographical references and index.

ISBN 978-0-387-95003-7 ISBN 978-1-4612-1178-5 (eBook)

DOI 10.1007/978-1-4612-1178-5

1. Medical education. 2. Medicine-Study and teaching (Residency). I. Title: How to compete successfully in the residency match program. II. Title.

[DNLM: 1. Education, Medical, Graduate-United States. 2. Internship and Residency_United States. W20 S658f 2000]

R737.S5767 2000

$610^{\circ} .71^{\circ} 55-\mathrm{dc} 21$

00-024951

Printed on acid-free paper.

\section{(c) 2000 Springer Science+Business Media New York}

Originally published by Springer-Verlag New York, Inc. in 2000

All rights reserved. This work may not be translated or copied in whole or in part without the written permission of the publisher, Springer Science+Business Media, LLC,

except for brief excerpts in connection with reviews or scholarly analysis. Use in

connection with any form of information storage and retrieval, electronic adaptation, computer software, or by similar or dissimilar methodology now known or hereafter developed is forbidden.

The use of general descriptive names, trade names, trademarks, etc., in this publication, even if the former are not especially identified, is not to be taken as a sign that such names, as understood by the Trade Marks and Merchandise Marks Act, may accordingly be used freely by anyone.

While the advice and information in this book are believed to be true and accurate at the date of going to press, neither the author nor the publisher can accept any legal responsibility for any errors or omissions that may be made. The publisher makes no warranty, express or implied, with respect to the material contained herein.

Neither the publisher nor the author makes any warranties, express or implied, as to results to be obtained from the use of online information derived from the websites provided in this book, including the warranties of merchantability and fitness for a particular use.

Neither Springer-Verlag nor the author shall assume any legal liability or responsibility for the accuracy, completeness, or usefulness, or any information, apparatus, product, or process disclosed herein (online or otherwise).

Reference to any specific professional organization, commercial product, process, or service by trade name, trademark, manufacturer, or otherwise does not imply its endorsement, recommendation, or favoring by Springer-Verlag. The views and opinions of the author expressed herein do not necessarily state or reflect those of Springer-Verlag.

Production managed by MaryAnn Brickner; manufacturing supervised by Jerome Basma. Photocomposed copy prepared from the author's Microsoft Word files.

9876554321

Additional material to this book can be downloaded from http://extras.springer.com. 


\section{Preface}

Medical graduates seeking training in the United States get their residency assignment through a matching process managed by the National Residency Matching Program (NRMP). The process is intimidating for most; traumatic for some. However, there are tips that can make the task easier and increase the likelihood of success. Many of these tips are known to those of us who advise students or are involved in the resident selection process, but this only helps if students have access to a good advisor, and not everyone does. This text is designed to fill that need for the student without access and to supplement the advice received for those who do.

No text can provide final answers to questions that are inherently subjective-only you, the student, can pose and answer those questions. The intent of this text is to help you frame those questions, develop strategies to assess them, and, finally, feel comfortable with your final decision. This book provides a glimpse into what residencies look for, how to choose the right program for yourself, and the sequence of tasks you must accomplish to navigate the process. It is easy to believe that everyone else is better, faster, bigger, and after exactly the spot you want. This book gives you the tools you need to ensure that you can be anybody's match. 


\section{Contents}

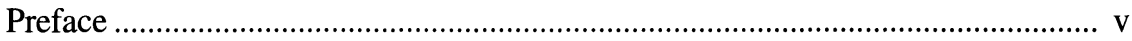

Chapter 1 Graduate Medical Education and "The Match".................................... 1

Anybody's Match........................................................................................ 1

Postgraduate Education................................................................................... 2

The Application Process .................................................................................. 4

Types of Residencies..................................................................................... 5

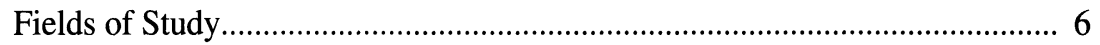

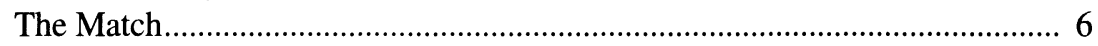

Chapter 2 Choosing Residencies ....................................................................... 9

Styles and Contents .................................................................................... 9

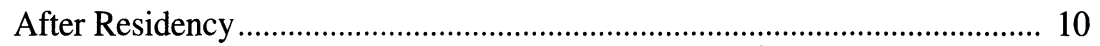

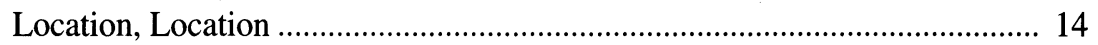

Competitiveness ..................................................................................... 15

Does Size Really Matter?........................................................................... 16

The Myth of "The" Residency........................................................................... 17

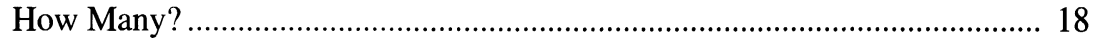

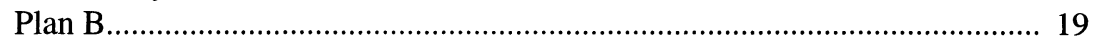

Chapter 3 Choosing Residents; Choosing You ..................................................... 23

Who Are They Looking For?...................................................................... 23

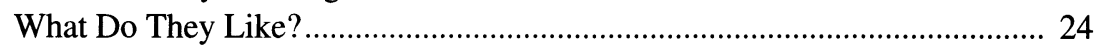

Factors That Get You Noticed ...................................................................... 25

How They Carry Out the Process ...................................................................... 25

Chapter 4 The Master Timetable ......................................................................... 29

What Has to Be Done?.......................................................................... 29

Milestones to Shoot for .......................................................................... 30

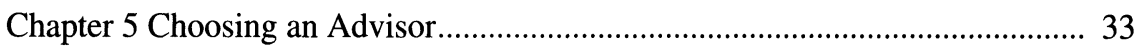

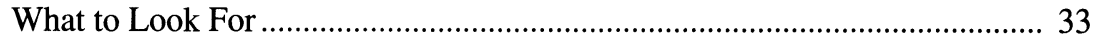

What You Don't Need .............................................................................. 35

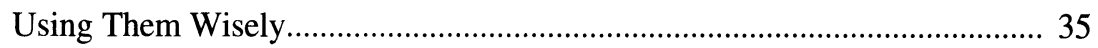




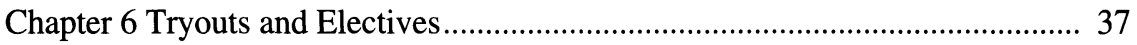

Choosing Senior Electives ...................................................................... 37

Electives as "Wild Hairs" ........................................................................ 39

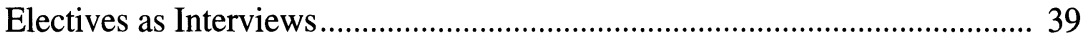

Chapter 7 Making the List_-Your First Cut ..................................................... 41

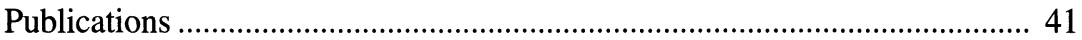

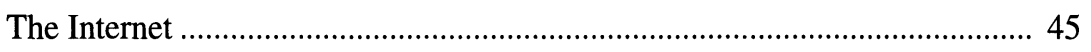

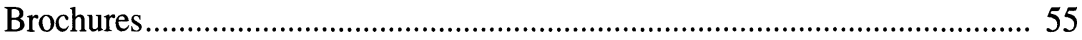

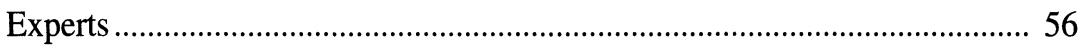

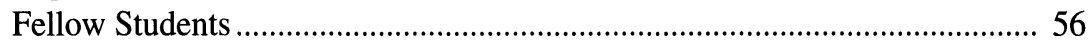

Chapter 8 Application Forms........................................................................ 59

Paper or Plastic? .................................................................................... 59

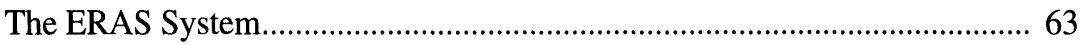

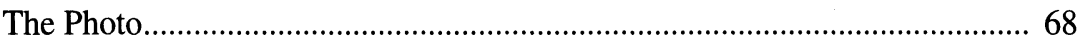

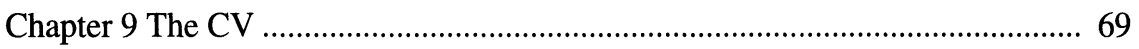

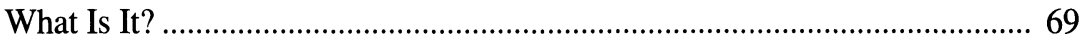

The Effective CV ..................................................................................... 70

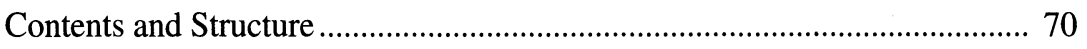

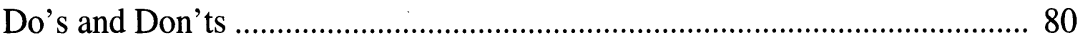

Chapter 10 Effective Personal Statements ..................................................... 83

Why the Personal Statement? ............................................................... 83

The Painful Process ....................................................................................... 84

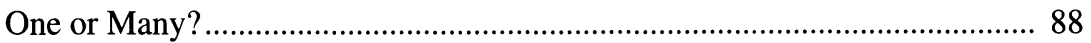

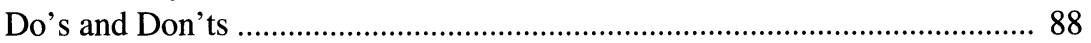

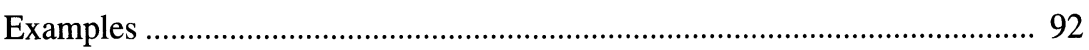

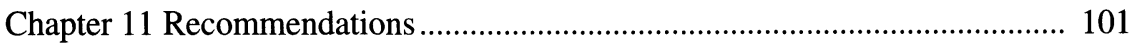

Who to Pick? ...................................................................................... 101

What to Ask Them For?............................................................................ 103

The Dean's Letter................................................................................. 105

Final Hints ................................................................................... 105

Chapter 12 Making the List-Theirs (The Interview and After) .................... 107

Getting the Slot......................................................................................... 107

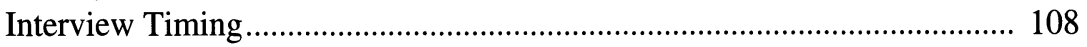

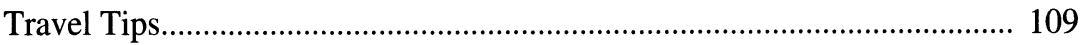

The Required Suit (Clothes and Grooming) …........................................ 110

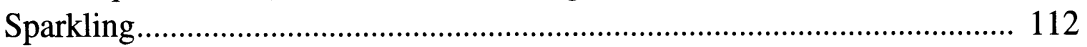

Fielding Questions ............................................................................ 112

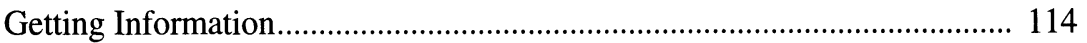




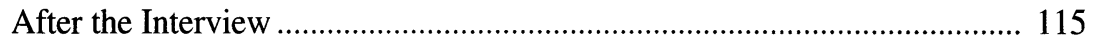

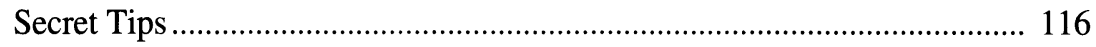

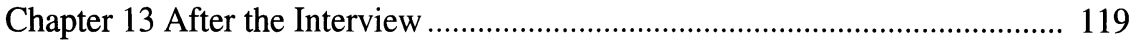

What They Do After You Leave ………………………........................... 119

Notes to Yourself ................................................................................ 120

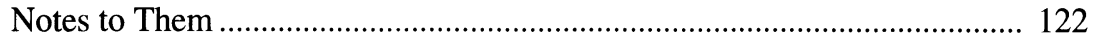

After the Last Interview .......................................................................... 126

Chapter 14 Making the List_-Your Match List............................................... 127

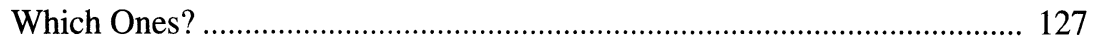

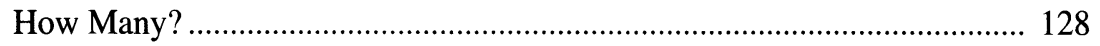

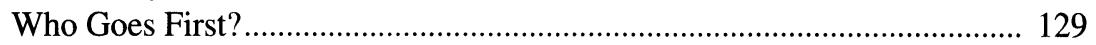

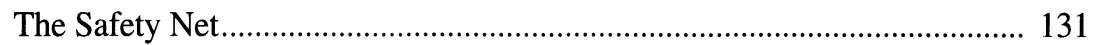

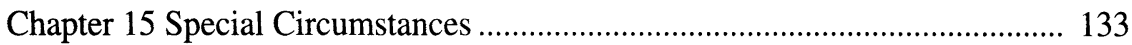

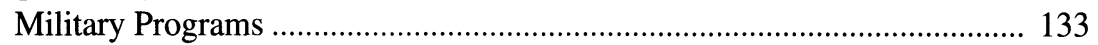

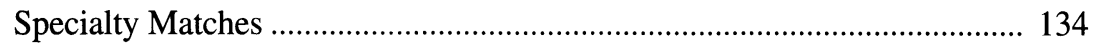

Osteopathic Programs …………………………………................... 140

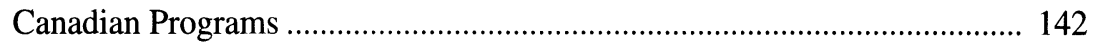

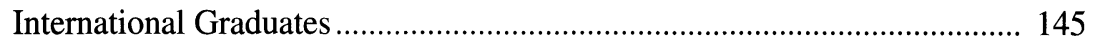

The Couple's Match .......................................................................... 146

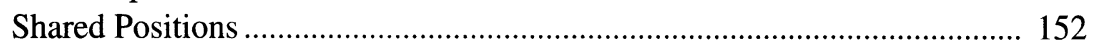

Special Needs ................................................................................. 152

Chapter 16 The Computer Match Process...................................................... 155

Who Participates? .................................................................................. 155

What Does the Match Do? .......................................................................... 156

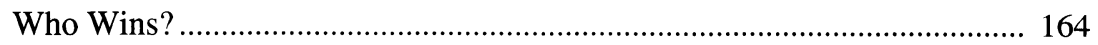

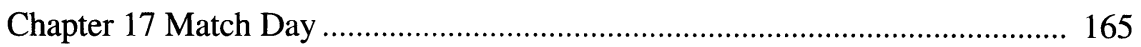

What Happens?

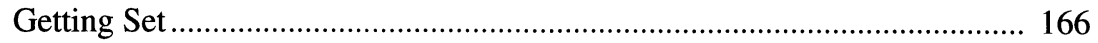

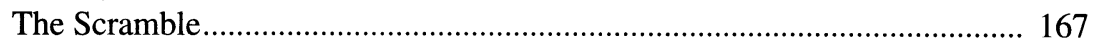

Chapter 18 Resources and References ......................................................... 171

Resources for Further Information ............................................................ 171

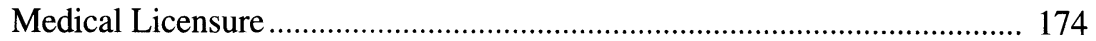

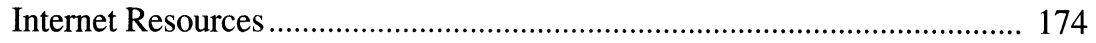

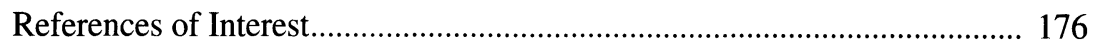

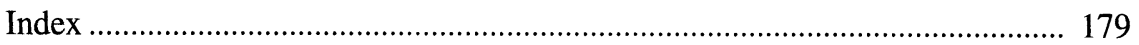

